
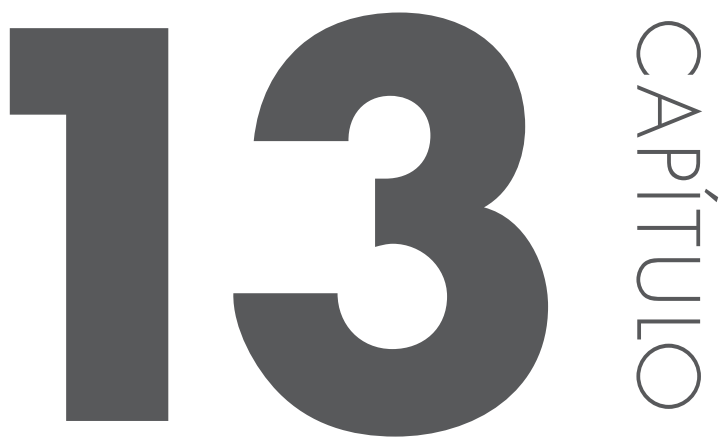

\title{
A EVOLUC̣ÃO HISTÓRICA E OS MESTRES DA QUALIDADE
}

Rodrigues, Bruno Felipe da Silva ${ }^{1}$;

Bachega, Stella Jacyszyn '

${ }^{1}$ Universidade Federal de Goiás - Regional de Catalão

* email: brunofelipesr@hotmail.com 
Resumo: A evolução da qualidade no decorrer da história passou por diferentes fases. Atualmente o conceito de qualidade é utilizado sobre diversas situações e contextos, estando difundida por todas as áreas do conhecimento e ambientes corporativos. Vários teóricos tiveram papel relevante na construção dos conceitos relacionados à área, auxiliando no desenvolvimento de diversas técnicas, metodologias e ferramentas de qualidade. $O$ objetivo deste artigo é explanar sobre as principais fases da evolução da qualidade, destacando suas principais características e contribuições, além de elencar os principais pensadores da qualidade e suas teorias. Para tanto, foi utilizado o método de pesquisa teóricoconceitual. Os seguintes mestres da qualidade foram discutidos: Walter A. Shewhart, William Edwards Deming, Joseph M. Juran, Philip B. Crosby, Armand V. Feigenbaum, Kaoru Ishikawa, e Genichi Taguchi. Dentre os principais fatos observados nas grandes eras da qualidade salientam-se: a implementação de tolerâncias, controle dimensional e padronização de produtos, e a diminuição da variabilidade dos sistemas de produção através da inserção de métodos estatísticos.

\section{Palavras-chave: Qualidade; Evolução histórica; Pesquisa teórico-conceitual}

Rodrigues, Bruno Felipe da Silva; Bachega, Stella Jacyszyn; "A EVOLUÇÃO HISTÓRICA E OS MESTRES DA QUALIDADE", p.

200-217 . In: Seminário de Pesquisa, Pós-Graduação e Inovação da Regional Catalão (2. : 2014 : Goiás) Coletânea

Interdisciplinar em Pesquisa, Pós-Graduação e Inovação - Volume 3 : Engenharias, Saúde e Gestão. Anais [livro eletrônico] / organizado por Adriana Freitas Neves, Idelvone Mendes Ferreira, Maria Helena de Paula, Petrus Henrique Ribeiro dos Anjos. São Paulo: Blucher, 2015. ISBN: 978-85-8039-113-8, DOI 10.5151/9788580391138-V3_Cap13 


\section{Introdução}

O conceito de qualidade é utilizado atualmente sobre diversas situações e contextos, estando difundida por todas as áreas do conhecimento e ambientes corporativos. No entanto, a grande abrangência do termo e sua expansão pelos diversos setores da economia teve sua motivação encontrada na evolução histórica da qualidade, que sempre acompanhou o desenvolvimento das necessidades humanas na busca por melhor competitividade e excelência em suas atividades (ROCHA, 2009).

A evolução da qualidade no decorrer da história passou por diferentes fases. Os estudos, conceitos e teorias desenvolvidos em cada uma dessas fases trouxeram 
muitas contribuições para o processo de construção do conceito de qualidade e acabaram se tornando parte fundamental para levar a Gestão da Qualidade a ser considerada como uma perspectiva estratégica que, segundo Carvalho $(2012$, p. 27), "não é vista de forma isolada, mas inserida em um modelo em que se consideram os aspectos essenciais de sobrevivência da organização".

Nesse sentido, diversos teóricos tiveram papel relevante na construção dos conceitos relacionados à área, auxiliando no desenvolvimento de diversas técnicas, metodologias e ferramentas de qualidade. As pesquisas desenvolvidas por alguns estudiosos trouxeram contribuições que se tornaram fatores determinantes de sucesso para diversas instituições, o que acabou trazendo o reconhecimento dos mesmos através da denominação de Mestres ou Gurus da Qualidade (CARVALHO, 2012).

Assim, a importância desta pesquisa vem da necessidade de se demonstrar a toda comunidade acadêmica o quão é importante estudar a evolução histórica da qualidade para se entender como os conceitos e abordagens atuais foram alcançados. Logo, o objetivo deste artigo é explanar sobre as principais fases da evolução da qualidade, destacando suas principais características e contribuições, além de elencar os principais pensadores da qualidade e suas teorias.

O presente artigo está estruturado da seguinte maneira: na próxima seção é apresentada a metodologia de pesquisa; na terceira seção é demonstrada a evolução histórica da qualidade; em seguida, são apresentados os principais mestres da qualidade; por último, a quinta seção apresenta as considerações finais.

\section{Método de pesquisa}

A pesquisa teórico-conceitual pode ser considerada como uma revisão da literatura onde um levantamento bibliográfico é desenvolvido com o intuito de identificar teorias que possam ser utilizadas como bases de um novo estudo (HEERDT; LEONEL, 2007). Além disso, este tipo de pesquisa possui o objetivo de explicar um problema a partir de referências teóricas publicadas em documentos, podendo ser efetuada como parte da pesquisa descritiva ou experimental ou, ainda, de forma independente (SILVA; SILVA, 2004).

De acordo com Berto e Nakano (2000), esse tipo de pesquisa é fruto de uma série de reflexões fundamentadas em um fato observado ou exposto pela literatura, reunião de opiniões e ideias de diversos autores ou mesmo pela simulação e modelagem teórica. Conforme esses autores, as discussões conceituais baseadas na literatura e revisões bibliográficas são classificadas como pesquisas teórico-conceituais. Neste trabalho foi realizada a pesquisa teórico-conceitual, ou bibliográfica, com o intuito 
de pré-orientação teórica a respeito da evolução da qualidade.

\section{Evoluc̣ão histórica da qualidade}

A qualidade, seus conceitos e práticas passaram por uma evolução bastante complexa ao longo da história. Garvin (2002) propõe uma classificação temporal que divide essa transformação em quatro eras, a saber: Inspeção, Controle Estatístico do Processo, Garantia da Qualidade e Gestão da Qualidade Total.

Até o final do século XIX, o sistema produtivo vigente era estritamente artesanal e baseado no conhecimento do fabricante. Nesse período, o artesão tinha o domínio completo de todo o sistema de produção, coordenando desde a fase de concepção do produto, escolha de matéria-prima, até as questões referentes ao pós-venda. Além disso, o controle de qualidade era realizado em todos os produtos pelo próprio artesão, sem uma preocupação maior sobre o processo de produção (STEVENSON, 2001).

Anãoexistêncianaépoca deumpadrãodeproduçãobem definidoimpossibilitava ao artesão pensar em questões como metrologia, controle dimensional, tolerâncias e especificações, resultando em produtos diferentes desenvolvidos sobre o mesmo projeto. Dessa maneira, as partes e peças dos produtos eram únicas e não eram criadas peças intercambiáveis (CARVALHO, 2012).

Com o evento da Revolução Industrial, um novo sistema de produção foi estabelecido, no qual foram implementadas a padronização dos produtos e a produção em larga escala. A escola taylorista da Administração Científica influenciou a especialização do trabalho, reduzindo as funções do trabalhador a apenas uma parte da produção, onde o mesmo realizava apenas uma atividade do processo, sem ter o domínio ou sequer o conhecimento sobre as outras etapas, além de não participar da concepção do produto e do planejamento (CARVALHO, 2012).

O controle da qualidade deixou de ser realizado durante todo o processo de produção e teve seu foco voltado à uniformidade do produto, ficando a verificação da qualidade realizada apenas no produto finalizado e sob a responsabilidade do inspetor, o que caracterizou a fase como a Era da Inspeção. Além disso, a linha de produção permitiu a produção em massa de produtos iguais, permitindo o surgimento dos primeiros sistemas de medição padronizados, bem como dos primeiros conceitos acerca de metrologia, especificações e tolerâncias. No entanto, não existia preocupação maior com a análise crítica e solução de problemas ou com as causas e consequências dos mesmos (BUENO, 2003).

A partir de 1924, a variabilidade dos processos de produção passou a ser reconhecida. A nova visão sobre as variações das matérias-primas, dos operários, 
dos equipamentos e de diversos outros fatores relacionados ao processo produtivo, condicionou Walter A. Shewhart desenvolver os gráficos ou cartas de controle, que facilitaram a inserção dos conceitos da estatística no sistema de produção. O Controle Estatístico da Qualidade foi desenvolvido com o objetivo de identificar as variações de processo que resultavam em problemas (VERAS, 2009).

A aplicação dos métodos estatísticos durante a Era do Controle permitiu que a qualidade passasse a ser controlada por amostragem, e a análise e solução de problemas se tornou menos complexa a partir do desenvolvimento de diversas técnicas e metodologias, como o ciclo PDCA (Plan, Do, Check, Action), proposto por Shewhart, e as sete ferramentas básicas da qualidade. Além disso, a fase é caracterizada pela criação das primeiras normas de controle estatístico da qualidade, como a britânica BS600 e a americana AWS Z1.1-Z1.3 (PALADINI, 2012).

Uma nova era acabou se estabelecendo, no período pós-Segunda Guerra Mundial, após a difusão dos modelos de controle estatístico: a Era da Garantia da Qualidade. Durante essa fase, o conceito de qualidade, impulsionado pelo crescimento do volume e da complexidade dos produtos, evoluiu de um método restrito de controle a um método de gerenciamento, tomado pelo objetivo principal de criar sistemas preocupados na prevenção e solução de problemas (VERAS, 2009).

A consolidação das áreas de qualidade nas organizações fez surgir as primeiras associações de profissionais do setor como a japonesa JUSE (Japan Union of Scientists and Engineers) e a americana ASQC (American Society for Quality Control), atualmente ASQ (American Society for Quality). Além disso, os sistemas de garantia da qualidade desenvolvidos possibilitaram a expansão da qualidade além dos limites da fábrica, integrando as organizações e seus fornecedores, e facilitando a solução de problemas (OLIVEIRA, 2003).

Durante a Era da Garantia, o estabelecimento da prevenção de problemas como objetivo principal foi possibilitado a partir do alinhamento entre a expansão dos métodos estatísticos e o surgimento de novos conceitos e técnicas de planejamento e controle gerenciais: a quantificação dos custos de qualidade, proposta por Juran em 1951; o programa Zero Defeito, desenvolvido por Crosby; a confiabilidade; e o sistema de Controle da Qualidade Total ou TQC (Total Quality Control), formulado por Feigenbaum (QUEIROZ, 1995).

À mesma época, o surgimento do TQC levou a um redirecionamento da qualidade do enfoque em adequação às especificações a uma abordagem mais ampla, que envolvia todos os setores do ambiente empresarial. Assim, o TQC possibilitou o início do planejamento, da organização e implementação de Sistemas Integrados de Controle de Qualidade, os quais controlam o produto desde o projeto, materiais, processos, produto final (STEVENSON, 2001).

No entanto, a criação de sistemas de produção que faziam a integração dos 
diferentes departamentos com foco na qualidade aconteceu através de diversas abordagens diferentes, com destaque para o enfoque japonês e o enfoque ocidental. O enfoque ocidental, utilizado pela ASQC, pregava a adequação dos produtos às especificações. Tal abordagem tinha por objetivo a implementação de manuais, procedimentos e registros de resultados que possibilitassem a padronização da produção e a prevenção de falhas. Além disso, todos os departamentos recebiam responsabilidades sobre a manutenção da qualidade, criando, portanto, um enfoque sistêmico que visava cumprir normas técnicas, regulamentações governamentais, códigos e leis (CARVALHO, 2012).

Por outro lado, a segunda vertente se destacou pela incorporação dos trabalhadores na manutenção da qualidade, ficando conhecido como CWQC (Company Wide Quality Control). O controle da qualidade por toda a empresa, proposto pelo enfoque japonês, pregava que toda a empresa deveria ser dirigida ao atendimentoàs expectativas do consumidor da maneira mais viável economicamente. Para tal, foi necessária a abrangência de aspectos relacionados a outros ramos do conhecimento além da engenharia - psicologia, sociologia, educação e economia - para a implementação de programas de motivação, conscientização, capacitação e interação do homem na busca por objetivos de qualidade comuns (CARVALHO, 2012).

OCWQC obteve grande destaque internacional pelo surgimento de novas teorias e conceitos de gestão, que viriam a ser tomadas como modelos mais eficientes do que aqueles estabelecidos. Através do enfoque japonês aconteceu o desenvolvimento do modelo Toyota de produção, idealizado por Taiichi Ohno. O novo sistema de produção ficou conhecido por produção enxuta ou lean manufacturing e influenciou a qualidade através da eliminação dos desperdícios de produção - conhecido como muda em japonês. Além disso, diversas outras técnicas e metodologias auxiliaram na difusão do novo modelo e, principalmente, na devolução ao trabalhador a responsabilidade sobre a qualidade, com destaque para: os conceitos de melhoria contínua, conhecido como kaizen; a seleção e desenvolvimento integrado de fornecedores ou keiretsu; o poka yoke, criação de dispositivos à prova de erros; e os Círculos de Controle da Qualidade - CCQ (CARVALHO, 2012).

A partir de 1987, a criação de normas regulamentadoras possibilitou a eliminação de grande parte dos auditores mantidos pelas instituições. No novo cenário, instituições independentes ficaram responsáveis pelos processos de certificação e auditoria de fornecedores, emitindo relatórios e certificados de qualidade. Dessa maneira, o estabelecimento de normas, como a ISO 9000 de Sistemas de Garantia da Qualidade e a QS 9000 para o setor automotivo, retirou a responsabilidade das organizações sobre o desenvolvimento integrado de parceiros (RIBEIRO NETO; TAVARES; HOFFMANN, 2008). 
De uma maneira geral, os selos de certificação dos modelos normativos indicavam e garantiam que os produtos fornecidos apresentassem a excelência em qualidade exigida, ao mesmo tempo em que possibilitavam às organizações aumentar a eficiência de seus processos através do redirecionamento do foco de seus esforços para outras questões de importância igualitária que estavam dentro de sua responsabilidade, como afirmado por Carvalho (2012, p. 6): "introduzindo elementos da gestão por processos, gestão por diretrizes e foco no cliente".

Posteriormente, o surgimento de novos modelos normativos, como a ISO 26000 de Responsabilidade Social e OHSAS 18000 de saúde e segurança ocupacional, de maneira integrada às normas anteriormente estabelecidas, bem como aos diversos setores da empresa fez surgir os Sistemas Integrados de Gestão (SIG), dando início a uma nova era: a Era da Gestão da Qualidade Total ou Era da Gestão Estratégica da Qualidade.

Na nova Era estabelecida, as organizações apresentam sistemas produtivos totalmente integrados sob o objetivo comum de desenvolver produtos que apresentem o nível de qualidade excelente. Além disso, o foco passa a ser totalmente na satisfação das necessidades do consumidor final, ressurgindo conceitos equivalentes ao período artesanal, como a customização de produto, desenvolvida aqui como uma customização em larga escala (RIBEIRO NETO; TAVARES; HOFFMANN, 2008).

A moderna Gestão da Qualidade caracteriza-se também pela utilização da qualidade pelas organizações como diferencial competitivo. Como defende Bueno (2003, p. 27) a Gestão da Qualidade Total deve ser considerada como "uma estratégia administrativa e que deve estar alinhada à estratégia de negocio da empresa". Portanto, atualmente, a manutenção de um nível de qualidade excelente acaba influenciando de maneira direta a evolução das organizações.

Por fim, vale destacar que a evolução da qualidade aconteceu de modo que os conceitos, métodos e técnicas de cada fase não deixaram de ser utilizados nas eras subsequentes. Na realidade, as diferentes abordagens acabaram incorporadas aos métodos emergentes com o intuito de melhorar continuamente a gestão das organizações. A evolução está representada na Figura 1.

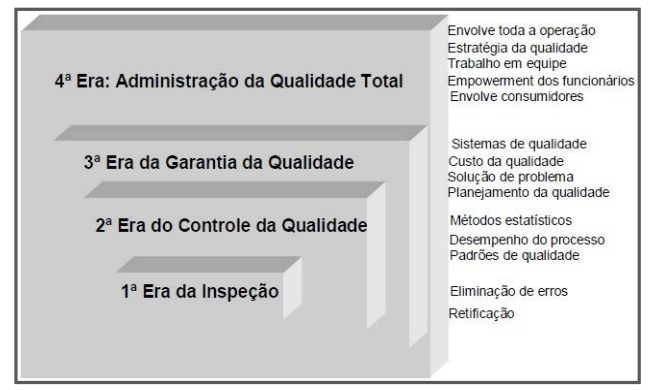

Figura 1 - A evolução histórica da qualidade.

Fonte: Adaptado de Barçante (1998). 


\section{Os Mestres da Qualidade}

Nessa seção são abordados os seguintes mestres da qualidade: Walter A. Shewhart, William Edwards Deming, Joseph M. Juran, Philip B. Crosby, Armand V. Feigenbaum, Kaoru Ishikawa, e Genichi Taguchi.

\subsection{Walter A. Shewhart}

Walter Shewhart nasceu nos Estados Unidos, em 1891. Engenheiro e doutor em física pela Universidade da Califórnia estabeleceu sua contribuição para a gestão da qualidade, principalmente, pelo trabalho desenvolvido na Western Eletric e na Bell Telephone Laboratories.

Shewhart ficou conhecido como o pai do controle estatístico da qualidade pelo desenvolvimento dos gráficos de controle do processo. Através do Controle Estatístico do Processo (CEP), o autor conseguiu adaptar os conceitos de estatística ao ambiente organizacional, formulando uma ferramenta gráfica que possibilitava distinguir de maneira clara os desvios ocasionados por variações comuns dos processos - causas normais - daquelas causas especiais, que não eram inerentes aos processos. Dessa maneira, a identificação, análise e solução de problemas passaram de uma abordagem reativa a um foco proativo de previsão (CARVALHO, 2012).

Além disso, Shewhart foi o responsável pelo desenvolvimento de uma metodologia de direcionamento da análise e solução de problemas que viria a se tornar conhecida com Edwards Deming: o ciclo PDCA (Plan, Do, Check, Action). O método propunha implementar a melhoria contínua através de planejamento, ações efetivas, verificação e ações de controle e correção.

\subsection{William Edwards Deming}

Edwards Deming, nascido nos Estados Unidos em 1900, era um consultor formado em engenharia elétrica e doutor em matemática e física pela Universidade de Yale. Apesar de se tornar discípulo de Walter A. Shewhart, devido ao grande interesse pelas ferramentas de controle estatístico, foi através do trabalho desenvolvido no Japão após a Segunda Guerra Mundial, principalmente como consultor da JUSE, que desenvolveu suas contribuições para a qualidade.

Deming defendia que a gestão da qualidade deveria utilizar uma abordagem na qual os métodos estatísticos seriam utilizados como ferramentas que possibilitariam a identificação e controle contínuos das variabilidades dos processos. A ideia 
ganhou força ao perceber que a participação dos trabalhadores, utilizada na cultura empresarial japonesa, na melhoria contínua da organização - o chamado kaizen $\urcorner$ era descrita de maneira sistematizada no método do ciclo PDCA criado por Shewhart (VERAS, 2009).

Além de ser o difusor do ciclo PDCA - utilizado como um plano de sistematização para a análise e solução de problemas - Deming proporcionou grande contribuição ao enunciar 14 princípios que deveriam ser seguidos para alcançar a excelência em gestão da qualidade. São eles:

1- Criar uma constância de propósitos para a melhoria de produtos e serviços, buscando manter a empresa competitiva, para se permanecer no mercado e gerar empregos;

2- Adotar uma nova filosofia, visto que os métodos de gestão ficam obsoletos e necessitam, portanto de transformação;

3- Acabar com a dependência da inspeção para alcançar a qualidade, investindo diretamente na prevenção e eliminação de defeitos do produto em si, através da introdução de ferramentas de qualidade;

4- Eliminar a prática de basear o negócio apenas no preço. Utilizar o pensamento de minimizar o custo total, estabelecendo relacionamento de longo prazo com um único fornecedor, baseado em qualidade e confiança;

5-Melhorar constante e continuamente o sistema de produção, para aprimorar a qualidade e a produtividade ao mesmo tempo em que reduz os custos;

6-Estabelecer treinamento no trabalho, utilizando métodos modernos de formação;

7-Estabelecer liderança, para ajudar as pessoas a desenvolver seu trabalho de uma melhor maneira;

8-Eliminar o medo através de um método de gestão que proporcione à liberdade às pessoas trabalharem de maneira efetiva;

9-Quebrar barreiras entre departamentos. Os diversos setores da empresa devem trabalhar de maneira conjunta, com uma comunicação eficiente;

10-Eliminar slogans, exortações e metas para os trabalhadores;

11-Não utilizar gestão por objetivos ou metas baseadas em indicadores quantitativos. Tais métodos prezam pela quantidade e não pela qualidade;

12-Remover barreiras que impeçam o trabalhador de sentir orgulho pelo que faz; 13-Instituir um programa de rigoroso de educação e aperfeiçoamento;

14-Envolver toda a organização no objetivo de alcançar a transformação. A transformação é tarefa de todos. 


\subsection{Joseph M. Juran}

Joseph M. Juran nasceu em 1904, na cidade de Braila, na Romênia. Mudou-se para os Estados Unidos, onde faz graduação em engenharia e direito. A carreira como consultor em gestão da qualidade iniciou-se no departamento de estatística da empresa Western Eletric, mas foi pelo trabalho desenvolvido no período pós-guerra no Japão que alcançou a notoriedade.

O conceito de qualidade proposto por Juran, defendendo que se o produto deve ser adequado ao seu uso, traz a ideia de que as características dos produtos deveriam apresentar aquilo que fosse exigido pelo usuário. Para isso, os processos deveriam ser controlados de maneira a não apresentar deficiências. $O$ autor sugeria, portanto, que toda a empresa deveria estar envolvida com a qualidade, ou seja, a responsabilidade pela satisfação do consumidor estaria do nível operacional ao nível tático (ROCHA, 2009).

Dentre as principais contribuições de Juran, vale destacar a chamada Trilogia da Qualidade, na qual o autor defende a ideia de que a gestão da qualidade divide-se em três pontos essenciais: planejamento, controle e melhoria. O planejamento da qualidade se faz necessário para identificar os consumidores e suas necessidades, estabelecer os objetivos de desempenho e os planos de ação para atendê-los. O controle da qualidade visa comparar o desempenho atual do sistema com os objetivos estabelecidos e corrigir os desvios encontrados. Por fim, a melhoria da qualidade visa identificar os pontos onde é possível melhorar o desempenho do sistema e desenvolver práticas que possibilitem o aperfeiçoamento do mesmo (CARVALHO, 2012).

Por outro lado, Juran também se destacou pela melhoria do conceito de cliente, no qual defendia que qualquer pessoa em contato com o produto durante a sua produção era considerada um cliente interno, enquanto o cliente externo era aquele que se relaciona com o produto acabado. Além disso, o autor obteve notável contribuição na classificação dos custos de não fazer certo na primeira vez, ou seja, os custos da não qualidade, que foram classificados em falhas, prevenção e avaliação.

\subsection{Philip B. Crosby}

Nascido na cidade de Wheeling, no estado norte americano da Vírginia, Philip B. Crosby formou-se primeiramente em engenharia. Após anos de experiência em diversas empresas, foi com a fundação da Philip Crosby Associates (PCA) em 1979, que passou a atuar como consultor de gestão empresarial, dedicando-se exclusivamente para a área de qualidade. 
A experiência obtida nas empresas nas quais trabalhou auxiliou Crosby na construção de ideias a respeito do Zero Defeito. O conceito propunha que não existia a necessidade de o produto ser perfeito e, sim, que todos deveriam estar comprometidos em atender aos requisitos na primeira vez, ou seja, o fazer da maneira correta na primeira vez resultava em bom nível de qualidade. No entanto, existia a necessidade de se estabelecer uma filosofia de trabalho que exigisse a prevenção, a utilização dos custos de qualidade como ferramentas de gestão e a padronização das especificações (VERAS, 2009).

De maneira semelhante à Deming, o autor propunha 14 pontos que deveriam ser priorizados para se manter uma gestão da qualidade eficiente:

1- Certificar que a alta gerência está comprometida com a qualidade;

2- Formar equipes de melhoria da qualidade com representantes de cada departamento;

3- Introduzir indicadores de desempenho da qualidade para identificar onde há a necessidade de melhoria;

4- Avaliar os custos da não qualidade e utilizá-los como ferramentas de melhoria;

5- Conscientizar todos os trabalhadores a respeito da importância da qualidade;

6- Implantar ações corretivas de problemas, a partir dos resultados das etapas anteriores;

7- Desenvolver um planejamento para a implantação do programa Zero Defeito;

8- Distribuir responsabilidades entre os funcionários e treiná-los para a manutenção do programa de melhoria;

9- Instituir o dia do zero defeito, para difundir os conceitos do programa;

10- Definir objetivos a serem alcançados, para estabelecer metas de melhoria da qualidade;

11- Eliminar as causas dos erros, através do incentivo e facilitação da comunicação das dificuldades encontradas;

12- Reconhecer publicamente aqueles que atingem os objetivos traçados;

13- Estabelecer Círculos de Qualidade para a manutenção e o monitoramento do processo de melhoria;

14- Realizar todos os passos de maneira contínua e interminável para que o programa de melhoria não acabe. 


\subsection{Armand V. Feigenbaum}

Armand Feigenbaum nasceu em 1922, nos Estados Unidos. Formado em engenharia e doutor em ciências pelo Massachusetts Institute of Technology (MIT), ficou conhecido pela formulação do sistema de Controle Total da Qualidade (TQC), o qual desenvolveu uma abordagem sistêmica da qualidade dentro das organizações.

Feigenbaum (1961) definiu o TQC como "um sistema eficaz para integração dos esforços dos diversos grupos em uma organização, no desenvolvimento da qualidade, na manutenção e na melhoria da qualidade". Nessa concepção, a qualidade passa a ser um aspecto relacionado a todas às operações da organização, deixando de ser considerada apenas um como um atributo do produto.

Por outro lado, o TQC também propõe que os interesses e desejos consumidores como o ponto de partida para o estabelecimento da qualidade. A qualidade deixa de ser baseada apenas no atendimento às especificações, passando a ser introduzida no produto a partir das necessidades dos clientes. Dessa maneira, alguns aspectos que não eram anteriormente considerados no estabelecimento da qualidade, como as características de marketing, de engenharia de fabricação e de manutenção do produto e serviço, passam a ser condicionantes para a satisfação das expectativas dos consumidores (VERAS, 2009).

Assim, Feigenbaum define que a qualidade deve ser implementada como uma filosofia de gestão, onde o seu estabelecimento como objetivo principal da organização consolida o compromisso com a excelência em seus produtos e processos como seu compromisso. Além disso, a qualidade é determinada pelo consumidor, o que necessita o comprometimento da alta direção, a implantação de trabalho em grupo e o aumento do poder de decisão do trabalhador através da redução dos níveis hierárquicos.

\subsection{Kaoru Ishikawa}

Kaoru Ishikawa era um professor e consultor de empresas, nascido no Japão em 1915. Apesar de graduar-se em química pela Universidade de Tóquio, teve papel de grande destaque no desenvolvimento do modelo de gestão japonês através de contribuições à formulação do CWQC, que enfatizava a participação do ser humano na qualidade, diferenciando-o, portanto, do TQC de Feigenbaum.

Ishikawa propôs que as organizações deveriam estabelecer os chamados Círculos de Controle da Qualidade (CCQ), os quais trabalhadores voluntários formariam grupos focados na melhoria contínua e se reuniriam regularmente para analisar e solucionar problemas. Assim, os CCQ's se tornaram uma maneira de colocar em prática os conceitos do controle de qualidade total japonês, considerando que seu aspecto 
humano se apresentava no fato que os trabalhadores de todos os níveis e setores da empresa assumirem responsabilidade sobre a qualidade (VERAS, 2009).

Além disso, Ishikawa também foi muito importante na sistematização das sete ferramentas básicas da qualidade, as quais deveriam ser utilizadas de maneira constante pelos CCQ's para a análise e solução dos problemas. As ferramentas de controle da qualidade são: Análise de Pareto; Diagrama de Causa e Efeito ou Diagrama Espinha de Peixe; Histograma; Folhas de Controle; Diagramas de Escada; Gráficos de Controle; e Fluxos de Controle.

\subsection{Genichi Taguchi}

Genichi Taguchi nasceu no Japão, em 1924 e graduou-se em engenharia e estatística. A sua fama veio do fato de diferenciar-se dos outros mestres da qualidade através do foco de suas atividades de qualidade no projeto do produto e não na produção, o que chamou de controle de qualidade off-line.

Dentre as definições de qualidade propostas pelo autor, destaca-se aquela em que o Taguchi (1990) defende que a "qualidade é a diminuição das perdas geradas por um produto, desde a produção até seu uso pelos clientes". Nessa visão, a qualidade deve ser tratada desde o início do produto até seu destino final, ou seja, a melhoria contínua deve estar presentes desde a fase de projeto, o que resultará em uma menor variabilidade dos processos e, consequentemente, na redução de desperdícios.

Além disso, Taguchi propunha que a qualidade poderia ser mensurada através das perdas geradas para a sociedade, as quais deveriam ser estimadas em função do tempo de vida útil do produto. Assim, o atendimento às especificações possuía menor influência na qualidade final do que o desempenho e características apresentadas pelo produto, o que necessitava o monitoramento e controle das variabilidades (ROCHA, 2009).

\section{Considerações Finais}

Através da pesquisa teórico-conceitual desenvolvida foi apresentada a evolução histórica da qualidade, bem como as principais contribuições daqueles estudiosos considerados como mestres da área. Portanto, o objetivo proposto neste trabalho foi alcançado.

Dentre as principais contribuições para o conceito de qualidade identificados por meio da apresentação das quatro grandes eras da qualidade, destacam-se: a 
implementação de tolerâncias, controle dimensional e padronização de produtos, controlados através de departamentos de inspeção durante a Era da Inspeção; a redução da variabilidade dos sistemas de produção através da inserção de métodos estatísticos na Era do Controle Estatístico da Qualidade; a criação, desenvolvimento e implantação de programas, normas e sistemas padronizados de controle da qualidade, levando, durante a Era da Garantia, a responsabilidade pela qualidade do produto para toda a cadeia produtiva; e o estabelecimento da qualidade como estratégia e oportunidade de diferenciação frenteàs necessidades do consumidorna Era da Gestão da QualidadeTotal.

Além disso, foi possível perceber que os estudos desenvolvidos pelos mestres da qualidade aqui apresentados tiveram papel relevantes durante todas as fases de evolução do conceito de qualidade. Assim, vale ressaltar a contribuição de Shewhart para o controle estatístico da qualidade, os conceitos de solução de problemas e melhoria de sistemas propostos por Deming, Juran e Crosby, a implantação dos sistemas de qualidade padronizados defendidos por Feigenbaum e Ishikawa, além do controle da qualidade desde o projeto introduzido por Taguchi.

Dessa maneira, a presente pesquisa contribui quanto à explicitação da maneira pela qual aconteceu a evolução da qualidade, até que os conceitos e teorias utilizadas atualmente fossem estabelecidos. Além disso, a apresentação dos principais mestres da qualidade instiga estudos desenvolvidos sobre o tema qualidade não apenas nas organizações, mas também nos ambientes acadêmicos. 


\section{The Historical Evolution and the Masters of Quality}

Abstract: The quality evolution throughout the history has gone through different phases. Currently, the concept of quality is used for many situations and contexts and is widespread to all areas of knowledge and corporate environments. Many theorists had a relevant role in the construction of the concepts related to the field, assisting the development of several techniques, methodologies and tools of quality. The purpose of this article is to explain about the main phases of quality evolution, highlighting its main characteristics and contributions, besides to list the major quality thinkers and their theories. For both, it was used the method of theoreticalconceptual research. The following teachers of quality were discussed: Walter A. Shewhart, William Edwards Deming, Joseph M. Juran, Philip B. Crosby, Armand V. Feigenbaum, Kaoru Ishikawa, e Genichi Taguchi. Among the main facts observed throughout the great eras of quality should be highlighted: the implementation of tolerances, dimensional control and standardization of products, and the decrease of variability in production systems by the insertion of statistical methods,

Keywords: Quality; Historical evolution; Theorical-conceptual research.

\section{Referências bibliográficas}

BARÇANTE, L. C. Qualidade Total: uma visão brasileira, o impacto estratégico na universidade e na empresa. Rio de Janeiro: Campus, 1998.

BERTO, R. M. V. S.; NAKANO, D. N. Metodologia da pesquisa e a engenharia de produção. Produção. ano 5. v. 9, n 2, p. 65-75, jul. 2000. Disponível em: < http://www.scielo.br/pdf/ prod/v9n2/v9n2a05.pdf> Acesso em: 11 nov. 2014.

BUENO, M. Gestão Pela Qualidade Total: Uma Estratégia Administrativa. Um Tributo ao Mestre do Controle da Qualidade Total Kaoru Ishikawa. Revista do Centro do Ensino Superior de Catalão. Catalão, ano 5, n. 8, p. 23-55, 2003. Disponível em: <http://www.cesuc.br/revista/ed-3/ gest\%E3o_pela_qualidade_total.pdf> Acesso em: 17 jun. 2014. 
CARVALHO, M. M. Histórico da Gestão da Qualidade. In: CARVALHO, M. M.; PALADINI, E. P. (coord). Gestão da Qualidade: Teoria e Casos. 2 ed. Rio de Janeiro: Elsevier - ABEPRO, 2012.

FEIGENBAUM, A. V. Total Quality Control: engineering and management. New York: McGraw-Hill, $1961.627 \mathrm{p}$.

GARVIN, D. A. Gerenciando a Qualidade: a visão estratégica e competitiva. Rio de Janeiro: Qualitymark, 2002, p. 357.

HEERDT, M. L.; LEONEL, V. Metodologia Científica e da Pesquisa: livro didático. 5 ed. Palhoça: UnisulVisual, 2007, 266 p.

OLIVEIRA, O. J. Gestão da Qualidade: Introdução à História e Fundamentos. In. OLIVEIRA, O. J. (org). Gestão da Qualidade: Tópicos Avançados. Thomson, 2003, 243 p.

PALADINI, E. P. Ferramentas para a Gestão da Qualidade. In: CARVALHO, M. M.; PALADINI, E. P. (coord). Gestão da Qualidade: Teoria e Casos. 2 ed. Rio de Janeiro: Elsevier - ABEPRO, 2012.

QUEIROZ, E. K. R. de. Qualidade segundo Garvin. São Paulo: Annablume, 1995, 118 p.

RIBEIRO NETO, J. B. M.; TAVARES, J. da C.; HOFFMANN, S. C. Sistemas de Gestão Integrados: qualidade, meio ambiente, responsabilidade social e segurança e saúde no trabalho. São Paulo: Editora Senac São Paulo, 2008.

ROCHA, M. C. F. Gestão da Qualidade. Curitiba: IBPEX - ULBRA, 2009, 149 p.

SILVA, H. H. R.; SILVA, M. L. A. Metodologia da pesquisa. São Paulo: Salesiano, 2004.

STEVENSON, W. J. Administração das Operações de Produção. Rio de Janeiro: LTC, 2001.

TAGUCHI, G. Engenharia da Qualidade em Sistemas de Produção. São Paulo: McGraw-Hill, 1990.

VERAS, C. M. dos A. Gestão da Qualidade. São Luis: IFMA, 2009. 